\title{
Correction to: Persistent nepotism in peer-review
}

\author{
Ulf Sandström ${ }^{1} \cdot$ Martin Hällsten ${ }^{2}$
}

Published online: 17 October 2020

(c) Akadémiai Kiadó, Budapest, Hungary 2020

\section{Correction to: Scientometrics, Vol. 74, No. 2 (2008) 175-189 https://doi.org/10.1007/s11192-008-0211-3}

In our 2008 article, we have misinterpreted the interaction effects. As a result, we overestimate gender inequality.

On p. 186, we discuss gender differences in grades, and say that it is 9 percentage points higher for females, referring to model 9 of Table 2 . Here, the female dummy is interacted with three productivity measures. The coefficient of .096 refers to the case where all productivity measures are zero, which is outside the observed data range (compare Table 3). We erroneously ignore this when we interpret the gender coefficient and erroneously treat this coefficient as referring to average gender differences between males and females. A better estimate is model 8, which has no gender interactions (NB that these interactions are nonsignificant in model 9 and thus do not impact the model directly). Here, the estimate is instead .033, suggesting a weak average bonus for women.

This error also applies to model 10 and Table 1, and our main conclusions of the paper as discussed on p. 186 and also in the abstract. The interactions displayed in Table 1 ignore that gender and conflict of interest are interacted with the productivity measures, and thus refers to the situation where these are zero.

Using partial derivatives for gender:

$$
\begin{aligned}
\partial \text { score/ } \partial \mathrm{female} & =\beta_{\text {female }}+\beta_{\text {quality }(\mathrm{cpp} / \mathrm{fcs}) * \text { principal investigator is female }} * \text { quality }(\mathrm{cpp} / \mathrm{fcs}) \\
& +\beta_{\text {number of publications } * \text { principal investigator is female }} * \text { number of publications } \\
& +\beta_{\text {expected impact }(\mathrm{jcs} / \mathrm{fcs}) * \text { principal investigator is female }} * \text { expected impact }(\mathrm{jcs} / \mathrm{fcs}) \\
& +\beta_{\text {conflict of interest } * \text { principal investigator is female }} * \text { conflict of interest }
\end{aligned}
$$

The original article can be found online at https://doi.org/10.1007/s11192-008-0211-3.

Ulf Sandström

ulf.sandstrom@indek.kth.se

Martin Hällsten

martin.hallsten@sociology.su.se

1 Department of Industrial Economics and Management, KTH Royal Institute of Technology, Stockholm, Sweden

2 Department of Sociology, Stockholm University, Stockholm, Sweden 
we can re-center the estimates to reflect sample means. We collect the relevant coefficients from Table 2, model 10, and set the productivity measures to their sample means found Table 3. We then compute different margins for cases with and without conflict of interest.

$$
\begin{aligned}
& \text { Conflict of interest: } 0.097+-0.011 * 1.203+0.001 * 16.686 \\
& \quad+-0.041 * 1.128+-0.082 * 1=-.027795 \\
& \text { No association: } 0.097+-0.011 * 1.203+0.001 * 16.686 \\
& \quad+-0.041 * 1.128+-0.082 * 0=.054205
\end{aligned}
$$

These show that females have slight disadvantage when there is conflict of interest, but have an advantage when there is no conflict of interest. This in line with out conclusions, but the inequality is weaker.

Reanalyzing our data, we can also predict average marginal effects and predictions (using the margins command in stata) so we get inferential statistics. This is marginal effects and predicted values calculated for each observation in our data and then averaged, which also projects estimates back to our observed mean productivity and is representative of our data.

Figure E1 and Table E1 shows predicted values for each of the cells of our original Table 1. The pattern is the same as in our original article, but the differences by conflict of interest and gender are smaller. We find that men without contacts receive the lowest grading, and that there are no differences among the other categories as judged by the overlapping confidence intervals.

Table E2 displays the average marginal effect for gender for cases with and without reviewer affiliation. Precisely as with our calculations using partial derivatives above, we find the gender coefficient to be 0.05 when there is no reviewer affiliation, suggesting a disadvantage of 5 percentage points for men without contacts compared to women. This is half the size of the original effect we report. The difference is still highly

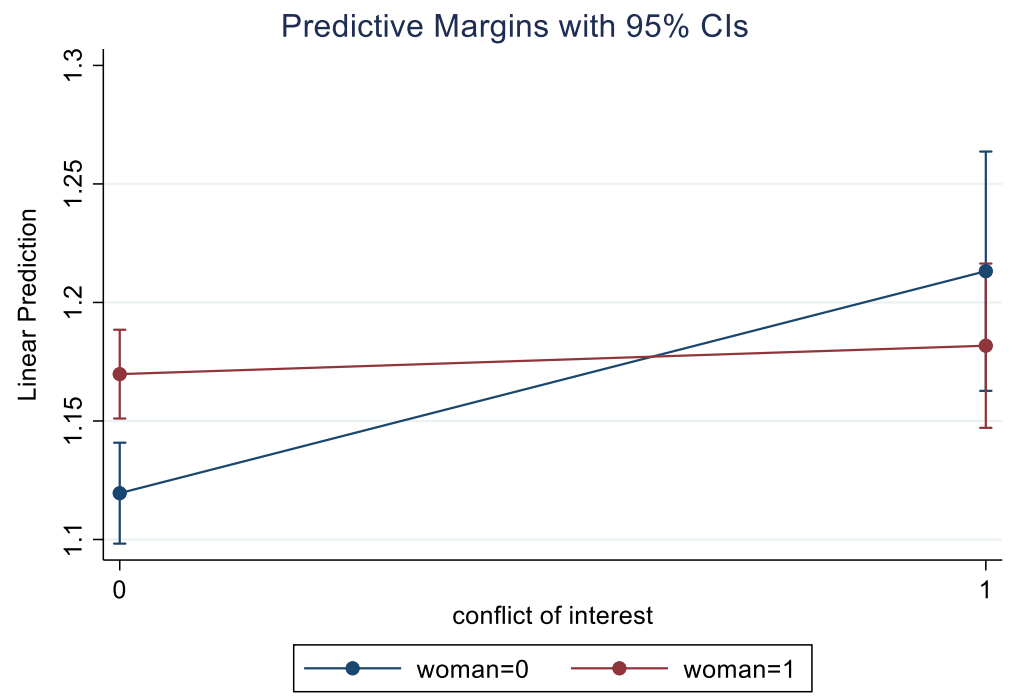

Fig. E1 Predicted grade scores by gender and conflict of interest, averaged over all cases 
Table E1 Predicted grade scores by gender and conflict of interest, averaged over all cases

\begin{tabular}{|c|c|c|c|c|}
\hline & \multicolumn{2}{|c|}{ Predicted grades } & \multicolumn{2}{|c|}{ Normalized to male, no CoI } \\
\hline & Male PI & Female PI & Male PI & Female PI \\
\hline No association & $\begin{array}{l}1.12 \\
(1.098,1.141)\end{array}$ & $\begin{array}{l}1.17 \\
(1.151,1.188)\end{array}$ & Ref. & 0.05 \\
\hline Conflict of interest & $\begin{array}{l}1.213 \\
(1.163,1.264)\end{array}$ & $\begin{array}{l}1.182 \\
(1.147,1.216)\end{array}$ & 0.093 & 0.062 \\
\hline
\end{tabular}

95\% CI in parenthesis. The normalization is scaled to be comparable to Table 1 in the original article

Table E2 Marginal effects of gender and conflict of interest, averaged over all cases

\begin{tabular}{lll}
\hline & \multicolumn{2}{l}{ Marginal effect of... } \\
\cline { 2 - 3 } & Female PI & Conflict of interest \\
\hline By factor... & & \\
COI & & \\
No association & $0.050^{* * * *}$ & \\
Conflict of interest & $(0.021,0.079)$ & \\
Gender & -0.031 & \\
Male PI & $(-0.096,0.033)$ & \\
Female PI & & $0.094^{* *}$ \\
& & $(0.037,0.150)$ \\
& & 0.012 \\
\end{tabular}

$95 \% \mathrm{CI}$ in parenthesis. ${ }^{*} p<0.05, * * p<0.01, * * * p<0.001$

significant. For males and females with reviewer affiliation, we find no significant difference (and the point estimate is close to the partial derivative above). If we change perspective to the marginal effect of conflict of interest, we find a reviewer bonus of almost ten percentage points for males, but no differences for females.

In sum, with correct interpretation, we now find weaker differences by conflict of interest and gender. The pattern we reported in the original article, namely that men without contacts receive the lowest grade scores, however still apply. We apologize to readers of our article for this error.

Acknowledgements We are grateful to Olle Folke at Department of Political Science at Uppsala University and SOFI at Stockholm University for pointing out this error in our work. 